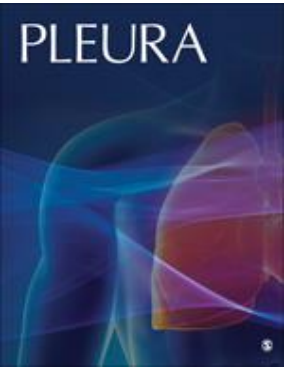

\title{
Review
}

\section{PD-L1 and Immunotherapy in Patients with Non-Small Cell Lung Cancer and Malignant Pleural Effusion}

Cole Liberator, $\mathrm{MD}^{1}$

Jonas Heymann, $\mathrm{MD}^{2}$

Anjali Saqi, MD, MBA ${ }^{2}$

Catherine Shu, MD ${ }^{1}$

John Crapanzano, $\mathrm{MD}^{2}$

William Bulman, $\mathrm{MD}^{1}$

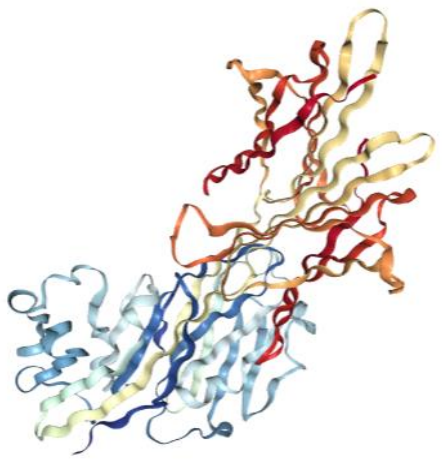

PD-1 and PD-L1 Complex

X-ray Diffraction

Courtesy RCSB Protein Data Bank Lin, D.Y. et al. PNAS USA (2008) 105 3011-3016 doi.org/10.1073/pnas.0712278105

Departments of ${ }^{1}$ Medicine and ${ }^{2}$ Pathology and Cell Biology, New York-Presbyterian Hospital-Columbia University Irving Medical Center, New York, NY

Corresponding author: Cole Liberator, MD, Division of Pulmonary, Allergy and Critical Care, Columbia University Irving Medical Center, 622 West $168^{\text {th }}$ Street, PH-840, New York, NY 10032

\section{$\underline{\text { Cd12142@cumc.columbia.edu }}$}

Funding support: This research received no specific grant from any funding agency in the public, commercial or not-for-profit sectors.

Conflict of interest disclosures: Catherine Shu has done an advisory board for Genentech. Anjali Saqi has a patent on a cell block device and has consulted for Boston Scientific. William Bulman is a consultant for Medtronic.

Abstract: Immunotherapy has emerged as a potent tool in the treatment of lung cancer, particularly in patients with advanced disease. Multiple drugs are now available which cause an anti-tumor immune response by blocking the interaction between programmed cell death protein 1 (PD-1) and its ligand, PDL1, which is expressed in some tumors. This review explores the role of immunotherapy and the practical implications of testing for PD-L1 in patients with malignant pleural effusion.

Key Words: PD-L1, immunotherapy, malignant pleural effusion, lung cancer 


\section{Introduction}

Dr. William Coley, fresh out of surgical residency in 1890, established a practice at the newly built New York Cancer Hospital on West $106^{\text {th }}$ Street and Eighth Avenue in Manhattan.

One of his first patients, seventeen year old Elizabeth Dashiell consulted him concerning a sarcoma on the dorsum of her hand. Her death following a futile arm amputation deeply affected the young surgeon. He researched hospital records and found the case of a German man who, after multiple procedures to remove a sarcoma from his cheek, developed wound erysipelas (Streptococcus pyogenes). The sarcoma gradually decreased in size, finally disappearing altogether, never to recur.

Dr. Coley theorized that a provoked immune system could

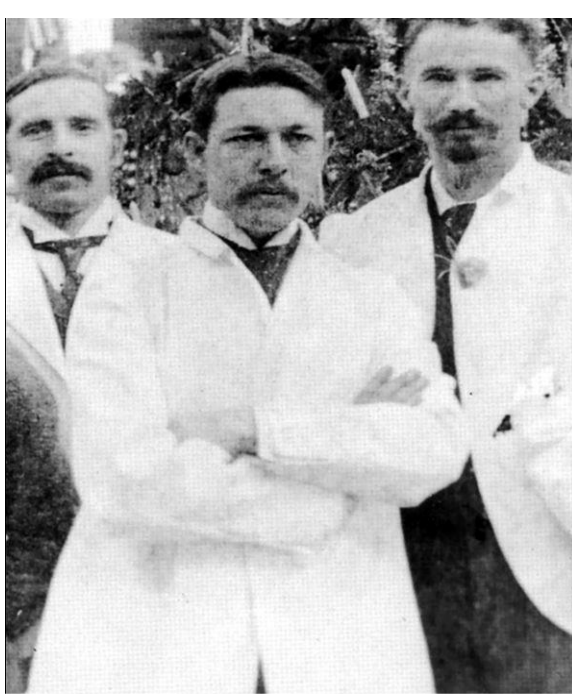

William Coley MD, Surgical Resident New York Hospital. Circa 1890
Malignant pleural effusion (MPE) is a complication seen with nearly all types of cancer. Lung cancer is the most common, with MPE found in nearly one third of patients. Malignant effusion is also commonly seen in breast cancer, Hodgkin's and Non-Hodgkin's lymphoma, and melanoma. ${ }^{2}$ Malignancy in an effusion results from hematologic spread, lymphatic spread, or direct invasion from peri-pleural disease. ${ }^{3-5}$ For solid tumors other than mesothelioma, malignant cells in the pleural space represent Stage 4 disease and poor survival. ${ }^{6}$ For patients with NSCLC, the most common cause of cancer-related death worldwide, ${ }^{7,8}$ the one-year survival rate for patients with Stage 4 disease on the basis of a malignant pleural effusion is engage in an anti-tumor immune response. He experimented with live and killed bacteria and bacterial toxins in patients with a variety of cancers, and published his work as a small case series. ${ }^{1}$ Interest in "Coley's Toxins" as a possible treatment modality was modest and short-lived, supplanted by the exciting development of radiation therapy.

Over a century later, we are witnessing a paradigm shift in cancer treatment that proves Dr. Coley to be prescient, at the very least. Cancer immunotherapy has emerged as an exciting new tool for fighting multiple types of cancer, particularly in patients with advanced disease. In this review, we examine the possible benefits and the limits of our knowledge in the realm of immunotherapy for patients with non-small cell $39 \%{ }^{9}$

In these patients, thoracentesis for pleural fluid cytological analysis is a safe, high yield bedside procedure to establish diagnosis, histologic subtype and disease stage in a single step. It has been shown to reliably identify targetable mutations and gene rearrangements. ${ }^{10-13}$ Similarly high yields are seen with thoracentesis in malignant pleural effusions from other solid cancers, including breast carcinoma $(70 \%) .{ }^{14}$ The yield for liquid tumors including lymphoma, leukemia and multiple myeloma ranges from 31$55 \%$, with Hodgkin's lymphoma generally having the lowest yield. ${ }^{15,16}$ 


\section{Systemic Treatment Options for Patients with Stage IV NSCLC and MPE}

A decade ago, treatment options for NSCLC Stage 4 on the basis of pleural involvement consisted of either palliative systemic chemotherapy or pleural interventions including thoracentesis, tunneled indwelling catheters, and pleurodesis. Today, several new treatment modalities have shown efficacy in selected patients. One is mutation-targeted therapy. Multiple genetic alterations that drive tumor formation and growth have been identified in a variety of tumors. Corresponding targeted therapies have been developed to prolong progression-free survival in patients with the corresponding genetic alterations.

Adenocarcinoma (ADC), the most common NSCLC subtype, contains activated epidermal growth factor receptor $(E G F R)$ mutations and re-arrangement of the anaplastic lymphoma kinase $(A L K)$. Drugs that target these and other mutations have been approved by the Food and Drug Administration (FDA) as first-line therapy for patients with advanced disease and are in wide clinical use. The treatment of metastatic cancer with these drugs therefore often hinges upon the detection of these genetic alterations. Despite the challenge frequently posed by the availability of only limited tissue, clinical tumor genotyping has been extensively validated on cytologic and small histologic specimens, including pleural fluid cytology. ${ }^{17-23}$ Accordingly, joint guidelines from the College of American Pathologists, International Association for the Study of Lung Cancer, and Association for Molecular Pathology, ${ }^{24}$ later endorsed by the American Society of Clinical Oncology(ASCO), ${ }^{25}$ support EGFR, ALK, ROS1 and BRAF (by AS$\mathrm{CO})$ testing on cytologic samples, including cell blocks prepared from pleural and pericardial effusion samples.

The newest option available for patients with locally advanced or metastatic disease, in- cluding those without a targetable genetic alteration in the tumor is immunotherapy. This latest approach shows that Dr. Coley was correct in his assertion that the immune system plays a role in cancer, but it is a complex one. Tumor cells express antigens that are targets for the immune system's cytotoxic anti-tumor response, a reaction conducted primarily by tumor specific cytotoxic T cells. An anti-tumor immune response can suppress tumor growth by eliminating cancer cells, but cancer cells, by evading immune surveillance, become free to proliferate. $^{26}$

\begin{tabular}{|l|}
\hline \multicolumn{1}{|c|}{ Treatment Options in NSCLC Stage 4} \\
\hline Mechanical Intervention \\
\hline Chemotherapy \\
\hline Targeted therapy against tumor mutations \\
\hline Immunotherapy to activate host defenses \\
\hline
\end{tabular}

At the beginning of this decade, it was shown that evasion of the immune system occurs by expression of ligands * on tumor cells that bind to and activate $\mathrm{T}$ cell inhibitory receptors. ${ }^{27}$ Multiple mechanisms for this evasion have been elucidated, including "immune checkpoints": biological pathways in the interaction between tumor cells and inflammatory cells in the tumor microenvironment. The most studied of these checkpoints is the pathway involving programmed cell death protein 1 (PD-1) and its ligands PD-L1 and PD-L2.

PD-1 is a receptor protein expressed primarily by $\mathrm{T}$ lymphocytes, including tumorinfiltrating $\mathrm{CD}^{+} \mathrm{T}$ cells, B cells, natural killer $\mathrm{T}$ cells, as well as by monocytes and dendritic cells. Binding of the PD-1 by tumor ligands inhibits the production (CHECK) of kinases involved in T cell activation. ${ }^{28}$ Thus, a tumor cell expressing PD-L1 can down-regulate the activity of tumor attacking $T$ cells. ${ }^{26}$ Converse- 
ly, prevention of this binding may enable infiltrating $\mathrm{T}$ cells to mount an immune attack on a cancer.

PD-1 has two ligands, PD-L1 and PD-L2, which are expressed by various tumors, including NSCLC, melanoma, breast carcinoma, renal cell carcinoma, ovarian carcinoma and gastrointestinal carcinoma. ${ }^{29-31}$ Upregulation PD-L1 on tumor cells has been shown to inhibit cytokine and $\mathrm{T}$ cell activation. ${ }^{29,32}$

In a landmark paper published in 2012, Brahmer and colleagues showed that a human monoclonal antibody specific for tumor PD-L1 given every 2 weeks for 12 weeks resulted in durable clinical responses in patients with a variety of cancers including 75 patients with advanced NSCLC who had failed or progressed on platinumbased therapy and/or tyrosine kinase inhibitors (TKIs). Benefit was seen in both adenocarcinoma (ADC) and squamous cell carcinoma (SCC). The drug was well tolerated. Complete or partial response was seen in up to $17 \%$ of patients. Prolonged stabilization of disease (at 24 weeks) was seen in up to $41 \% .^{33}$

Subsequent studies have focused specifically on patients with NSCLC. Several PD-1inhibitory monoclonal antibodies are now available to patients with metastatic NSCLC. ${ }^{34}$ Pembrolizumab was initially approved as a secondline treatment for both metastatic NSCLC with expression of PD-L1 in at least $50 \%$ of tumor cells, and for metastatic NSCLC that had progressed after platinum-based chemotherapy if PD-L1 expression was present in at least $1 \%$ of tumor cells. ${ }^{35-38}$ Pembrolizumab later gained
FDA accelerated approval as a first-line therapy in combination with chemotherapy for previously untreated patients with metastatic NSCLC. ${ }^{39}$ This approval is contingent on future survival data. Nivolumab was approved in 2015 for treatment of metastatic NSCLC in patients who had failed chemotherapy, and in patients with EGFR or ALK mutations who had failed targeted therapy.

Taken together, the efficacy of immunotherapy targeting the PD-1/PD-L1 axis in patients with metastatic lung cancer has been promising, with the potential to increase overall sur-

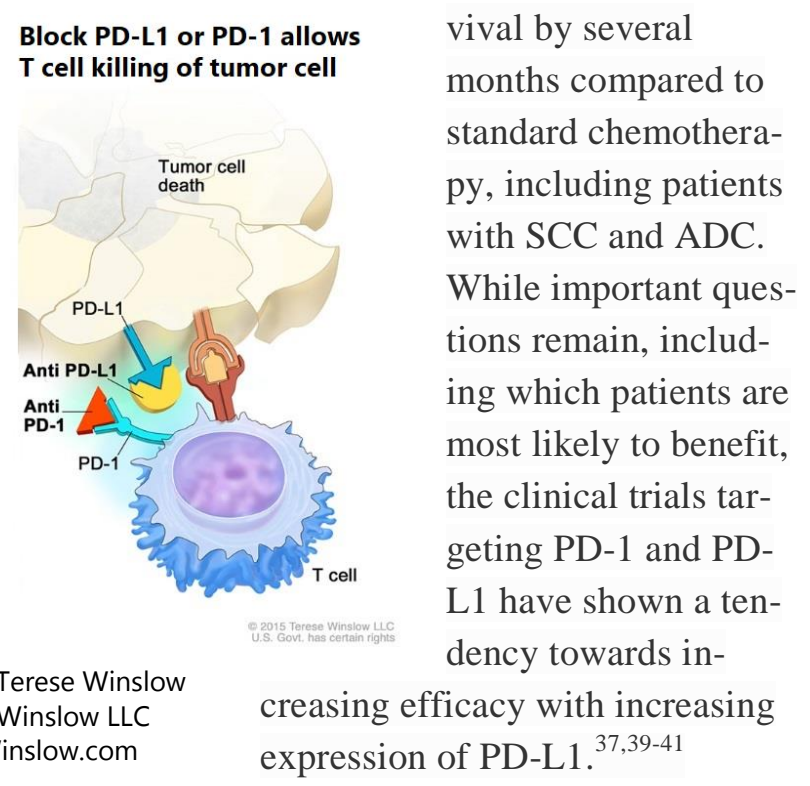

\section{PD-L1 Immunotherapy for Patients with NSCLC and MPE}

All of the large clinical trials of these agents in NSCLC required pathologic specimens. The PD-L1 tests were developed and validated on surgical specimens. The KEYNOTE-024 trial, for example, which led to first-line pembrolizumab approval, enrolled patients with tumors in which at least $50 \%$ of cells expressed PD-L1 in an immunohistochemistry (IHC)-based companion test (22C3 pharmDx - Dako North America, Inc., Carpinteria, CA). ${ }^{39}$ Although patients in the phase III trial of pembrolizumab as first-line therapy had unresectable, stage IV NSCLC, cy- 
tology specimens, including fine needle aspirates and effusion samples, were not permitted for quantification of PD-L1 expression under the trial protocol.

The stringent requirement for histology samples over cytology, along with the paucity of literature on PD-L1 in cytology samples, may lead clinicians and researchers to doubt the suitability of effusion samples for PD-L1 testing. The practical realities of clinical care, however, differ from those in a clinical trial. Roughly a third to half of all patients with NSCLC are diagnosed on the basis of a cytology specimen. ${ }^{42}$ For many patients with NSCLC staged on the basis of MPE, the pleural fluid tumor cells are the only cells easily available for PD-L1 testing.

Now that immunotherapy is available outside clinical trials, PD-L1 detection and quantification is routinely being done on tumor cells obtained from pleural and pericardial fluid samples in clinical practice. The information obtained is being used to guide clinical decision making, but there is a lack of literature validating this approach. Many questions remain open, chiefly whether the yield for PD-L1 testing in pleural fluid is the same as in histologic specimens, and whether the PD-L1 status of the pleural tumor cells is representative of the entire tumor burden. An analogous concern arises when testing small biopsy histologic samples from a large tumor. It is known that there is heterogeneity of PD-L1 expression in different regions of the same tumor. Therefore, a small piece may not represent the status of the whole. ${ }^{26,43}$
Pleural fluid for cytological analysis is prepared by spinning cells down received in either an alcohol-based fixative or $10 \%$ neutral buffered formalin (NBF) into a pellet known as a cell block. It is then processed like a histology sample - formalin fixed and paraffin embedded (FFPE). The suitability of cytologic specimens for other advanced testing, such as tumor mutation testing, has been well validated. Joint guidelines from the College of American Pathologists, the International Association for the Study of Lung Cancer and the Association for Molecular pathology all endorse the use of cytology for testing for EGFR and ALK. ${ }^{24}$ Theoretically, PDL1 testing should be easy and accurate in cytologic preparations. As currently employed, PD$\mathrm{L} 1$ analysis requires evaluation of a minimum of 100 viable tumor cells to be considered adequate for quantification of PD-L1 expression.

Three tests of PD-L1 expression by IHC are currently approved by the FDA, each with different grading scales and cut-offs for positivity. Variability between the assays may prove to be a large barrier to interpretation of biomarker data between trials. ${ }^{44}$ However, recent studies showing reasonable concordance between the assays are reassuring. ${ }^{40,45}$ Following correlation with a hematoxylin \& eosin-stained slide, a pathologist trained in scoring PD-L1 expression scores any perceptible membranous staining $(\geq 1+)$ of tumor cells and quantifies the proportion of viable, PDL1-expressing tumor cells in cytology and histology samples. Staining identified in necrotic cells or pulmonary alveolar macrophages is disregarded.

\section{Clinical Considerations \\ Pleural fluid cells may be the only ones easily available \\ PD-L1 testing of pleural fluid is done routinely \\ PD-L1 data used with paucity of literature support}

Obtaining other tissue can be a significant burden 
There is limited literature on the performance of PD-L1 testing in pleural fluid cytology. In the absence of clear data supporting PD-L1 testing in cytologic specimens, some have suggested that it may be necessary to perform more invasive sampling by core needle biopsy or surgical resection in patients with MPE. This approach, however, may be burdensome for patients with easily accessible tumor cells and advanced disease. As noted above, testing malignant cells processed from a pleural fluid sample for PD-L1 with IHC is technically quite feasible, ${ }^{46}$ the question remains whether these results are accurate and representative of (or concordant with) the malignant disease elsewhere.

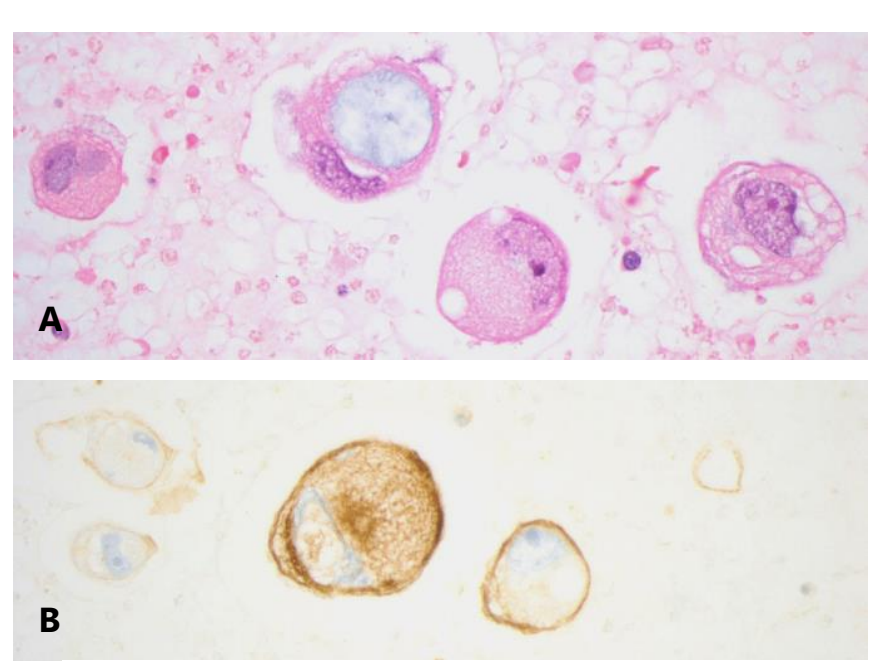

Figure: Pleural fluid collected by thoracentesis from a male smoker aged 60 years with suspected Stage 4 M1a primary lung adenocarcinoma.

(A) Pleural fluid cell block, $\mathrm{H} \& \mathrm{E}$; original magnification $\times$ 400. (B) Immunohistochemical analysis of PD-L1 expression demonstrating $1^{+}$membranous staining in approximately $75 \%$ of tumor cells ("PD-L1 positive").
In the Heymann study, a total of 214 lung carcinoma specimens were collected from 188 patients of median age 71. Pleural and pericardial effusion specimens from twelve of the patients were included. Specimens were deemed "positive" if $\geq 50 \%$ the viable tumor cells ex-

pressed PD-L1. Twenty-three patients had two or more specimens collected. PD-L1 expression was concordant among paired or triplicate samples from $21(91 \%)$ of these patients. Heymann and colleagues concluded overall that cytologic specimens of NSCLC provide sufficient cellularity for quantification of PD-L1 expression in a majority of cases, and that results of PD-L1 expression testing are comparable
There is some limited retrospective data to suggest that this is in fact the case, and little data to suggest that it is not.

Following FDA approval of immunotherapy for NSCLC, multiple groups have published retrospective analyses of PD-L1 testing in clinical practice These studies serve to inform in a limited way as to the suitability of MPE specimens for this purpose Heymann and colleagues published a series of consecutive cytologic specimens that were diagnostic of lung ADC and SCC and compared the results of PD-L1 quantification performed in a consecutive series of histologic specimens (both small biopsies and resections) collected over the same time period. $^{47}$ among resection, cytology, and other small biopsy specimens of NSCLC.

A case series published by Skov and Skov in 2017 had similar findings comparing NSCLC cytology to histology, although their series had only a small number of cytologic specimens from pleural fluid. Their retrospective review of 86 patients with paired histology and cytology specimens, mostly NSCLC and a few other thoracic neoplasms known to express PD-L1, showed similar rates of positivity across multiple cutoffs for "positive", from $\geq 1 \%$ to $\geq 50 \%$. Using the $\geq 50 \%$ cutoff, the paired specimens had $100 \%$ agreement on positive cases and $93 \%$ agreement on negative cases. Only three patients had a pleural fluid sample, making extrapolation 
of the findings overall to this small group difficult. $^{48}$

The largest cohort of pleural samples was recently presented in a case series by Ilie and colleagues. They performed a retrospective review of 70 paired biopsy and cytology samples from patients with NSCLC comparing PD-L1 expression across multiple assays Of those 70 cytological samples, 30 were from pleural effusions while the other 40 were from bronchial washings. Comparisons of PD-L1 tumor expression between biopsy and cytology showed strong correlation across multiple different assays and at both $1 \%$ and 50\% PD-L1 expression cut offs (> 95\% positive and negative agreement). Furthermore, intra-class correlation coefficients between isolated pleural cytology and biopsy were greater than 0.8 across various assays. These findings again support the viability of using PD-L1 expression in cytology samples to guide treatment eligibility for PD-L1 immunotherapy. $^{49}$

\section{Issues in PD-L1 testing in MPE}

Correlation of pleural fluid with histological specimens

Relationship between the pleural fluid yield and the whole tumor character

Comparison of three different PD-L1 tests.

Best cutoff for "PD-L1 positive"

Clinical application of limited data on pleural PD-L1 testing

\section{PD-L1 Expression in Other Malignancies with Pleural Invasion}

PD-L1 expression in the pleural fluid of patients with other primary malignancies has also been studied, most notably malignant pleural mesothelioma (MPM). Histological expression of PD-L1 in MPM is varied, ranging from 20$63 \%$ in limited studies. ${ }^{50,51}$ This wide range is attributed to different thresholds for PD-L1 positivity $(>1 \%$ to $>10 \%)$ as well as different percentages of MPM tumor subtypes between studies. Mansour and colleagues published a case series of PD-L1 expression in pleural effusions from 74 patients with MPM Of the analyzed cases, $10 \%$ had greater than $50 \%$ PD-L1 expression while $38 \%$ of the samples had greater than $1 \%$ expression. Of note, 13 of the 74 cases had to be excluded due to insufficient cell block material or malignant cell number.

\section{Conclusion}

The demonstration of a survival benefit in advanced NSCLC from mutation-targeted therapies and with PD-L1 immunotherapy has changed the way we approach the treatment of lung cancer. Many questions regarding PD-L1 testing remain under debate, including which test to use, whether to incorporate staining results of tumor-associated inflammatory cells, which cutoff to use for "positive", and what to do with negative tumors, given that some patients with PD-L1 negative tumors respond to therapy. A true measure of the utility of PD-L1 testing in pleural effusion samples would be a prospective trial with paired testing of malignant cells from pleural fluid and matched surgical specimens, with an analysis of clinical response in immunotherapy-treated patients. In light of the emerging data from case series, however limited, and given the increase in indications and popularity of PD-L1 inhibitors, the clinical equipoise for such a trial is evaporating. In the absence of such a trial, more data should be collected from patients who have paired samples collected in the course of routine clinical care.

In light of what we know now, quantification of PD-L1 expression in pleural fluid specimens of NSCLC is feasible. The results are comparable to histologic specimens in the majority of patients. For patients with stage IV dis- 
ease on the basis of a malignant effusion, PD-L1 testing on tumor cells from the pleural space is a low-risk, minimally invasive way to determine PD-L1 status. Existing data justify using this information to guide treatment decisions. For patients, the ultimate question is not one of PDL1 status, but of therapeutic benefit and prolongation of survival. Discordance in PD-L1 testing does occur, and testing can be suboptimal or fail for technical reasons. For patients with MPE and failed testing or equivocal results, or for those

PD-L1 negative patients with limited therapeutic alternatives, it is appropriate to consider resampling or testing other sites of disease.

\begin{abstract}
A Final Thought
We find ourselves now at the beginning of a new era of cancer treatment. We have learned much about the immune system's role in cancer prevention. And we have learned much about its limitations. We have shown that we can alter the interaction between tumor cells and normal cells. Yet, there is much to be learned and the work goes on. The final question remains to be answered. Can we enable the immune system to eradicate an established cancer?
\end{abstract}

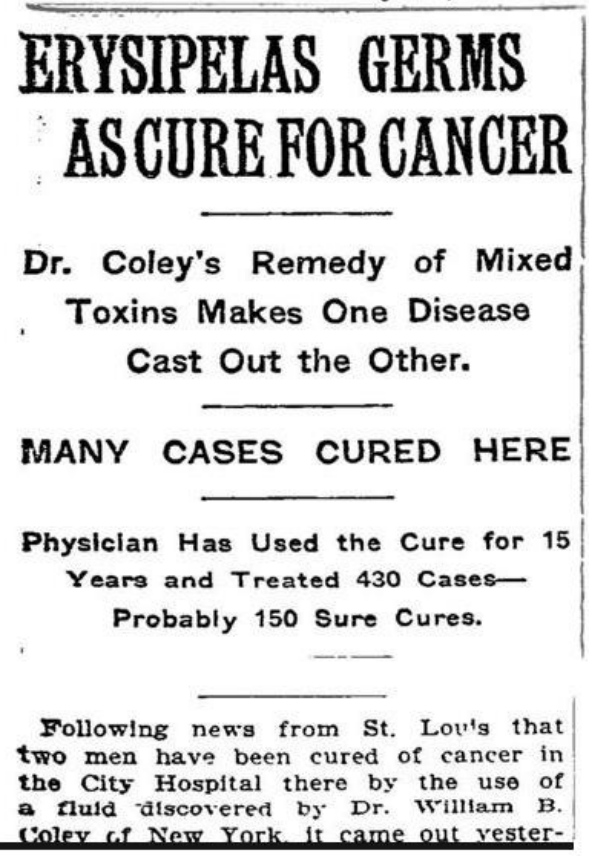

The authors express their gratitude to Jerome L. Slate MD, FCCP for his assistance in the preparation of this manuscript. 


\section{REFERENCES}

1. Coley WB, MD. The Classic: The Treatment of Malignant Tumors by Repeated Inoculations of Erysipelas: With a Report of Ten Original Cases. Clinical Orthopaedics \& Related Research January. 1991;262:3-11.

2. Management of Malignant Pleural Effusions. 2000;162(5):1987-2001.

3. Rodriguez-Panadero F, Naranjo FB, Mejias JL. Pleural metastatic tumours and effusions. Frequency and pathogenic mechanisms in a post-mortem series. European Respiratory Journal. 1989;2(4):366-369.

4. Chernow B, Sahn SA. Carcinomatous involvement of the pleura: an analysis of 96 patients. The American journal of medicine. 1977;63(5):695-702.

5. Andrews BS, Arora NS, Shadforth MF, Goldberg SK, Davis IV JS. The Role of Immune Complexes in the Pathogenesis of Pleural Effusions 1-3. American Review of Respiratory Disease. 1981;124(2):115-120.

6. Clive AO, Kahan BC, Hooper CE, et al. Predicting survival in malignant pleural effusion: development and validation of the LENT prognostic score. Thorax. 2014;69(12):1098-1104.

7. Siegel RL, Miller KD, Jemal A. Cancer statistics, 2016. CA Cancer J Clin. 2016;66(1):7-30.

8. Toh CK. The changing epidemiology of lung cancer. Methods Mol Biol. 2009;472:397-411.

9. Sánchez de Cos Escuín J, Abal Arca J, Melchor Íniguez R, et al. Tumor, node and metastasis classification of lung cancer - M1a versus M1b - Analysis of M descriptors and other prognostic factors. Lung Cancer. 2014;84(2):182-189.

10. Rivera MP, Mehta AC, Wahidi MM. Establishing the Diagnosis of Lung Cancer: Diagnosis and Management of Lung Cancer, 3rd ed: American College of Chest Physicians Evidence-Based Clinical Practice Guidelines. Chest. 2013;143(5, Supplement):e142S-e165S.

11. Barnes TW, Morgenthaler TI, Olson EJ, Hesley GK, Decker PA, Ryu JH. Sonographically guided thoracentesis and rate of pneumothorax. Journal of Clinical Ultrasound. 2005;33(9):442-446.

12. Bielsa S, Panadés MJ, Egido R, et al. Rentabilidad del estudio citológico del líquido pleural en el derrame maligno. Anales de Medicina Interna. 2008;25:173-177.

13. Hsu C. Cytologic detection of malignancy in pleural effusion: A review of 5,255 samples from 3,811 patients. Diagnostic Cytopathology. 1987;3(1):8-12.

14. Dines D, Pierre R, Franzen S. The value of cells in the pleural fluid in the differential diagnosis. Paper presented at: Mayo Clinic Proceedings1975.

15. Melamed MR. The cytological presentation of malignant lymphomas and related diseases in effusions. Cancer. 1963;16(4):413-431.

16. Whitcomb ME, Schwarz MI, Keller AR, Flannery EP, Blom J. Hodgkin's Disease of the Lung 1, 2. American Review of Respiratory Disease. 1972;106(1):79-85.

17. Rekhtman N, Brandt SM, Sigel CS, et al. Suitability of thoracic cytology for new therapeutic paradigms in non-small cell lung carcinoma: high accuracy of tumor subtyping and feasibility of EGFR and KRAS molecular testing. J Thorac Oncol. 2011;6(3):451-458.

18. Billah S, Stewart J, Staerkel G, Chen S, Gong Y, Guo M. EGFR and KRAS mutations in lung carcinoma: molecular testing by using cytology specimens. Cancer Cytopathol. 2011;119(2):111-117.

19. Heymann JJ, Bulman WA, Maxfield RA, et al. Molecular testing guidelines for lung adenocarcinoma: Utility of cell blocks and concordance between fine-needle aspiration cytology and histology samples. Cytojournal. 2014;11:12.

20. Coley SM, Crapanzano JP, Saqi A. FNA, core biopsy, or both for the diagnosis of lung carcinoma: Obtaining sufficient tissue for a specific diagnosis and molecular testing. Cancer Cytopathol. 2015;123(5):318-326.

21. DiBardino DM, Saqi A, Elvin JA, et al. Yield and Clinical Utility of Next-Generation Sequencing in Selected Patients With Lung Adenocarcinoma. Clin Lung Cancer. 2016;17(6):517-522. 
22. Roy-Chowdhuri S, Stewart J. Preanalytic Variables in Cytology: Lessons Learned From Next-Generation Sequencing-The MD Anderson Experience. Arch Pathol Lab Med. 2016;140(11):1191-1199.

23. Tian SK, Killian JK, Rekhtman N, et al. Optimizing Workflows and Processing of Cytologic Samples for Comprehensive Analysis by Next-Generation Sequencing: Memorial Sloan Kettering Cancer Center Experience. Arch Pathol Lab Med. 2016;140(11):1200-1205.

24. Lindeman NI, Cagle PT, Aisner DL, et al. Updated Molecular Testing Guideline for the Selection of Lung Cancer Patients for Treatment With Targeted Tyrosine Kinase Inhibitors: Guideline From the College of American Pathologists, the International Association for the Study of Lung Cancer, and the Association for Molecular Pathology. Journal of Thoracic Oncology. 2018;13(3):323-358.

25. Kalemkerian GP, Narula N, Kennedy EB, et al. Molecular Testing Guideline for the Selection of Patients With Lung Cancer for Treatment With Targeted Tyrosine Kinase Inhibitors: American Society of Clinical Oncology Endorsement of the College of American Pathologists/International Association for the Study of Lung Cancer/Association for Molecular Pathology Clinical Practice Guideline Update. Journal of Clinical Oncology. 2018:JCO.2017.2076.7293.

26. Ilie M, Hofman V, Dietel M, Soria J-C, Hofman P. Assessment of the PD-L1 status by immunohistochemistry: challenges and perspectives for therapeutic strategies in lung cancer patients. Virchows Archiv. 2016;468(5):511-525.

27. Mellman I, Coukos G, Dranoff G. Cancer immunotherapy comes of age. Nature. 2011;480:480.

28. Pardoll DM. The blockade of immune checkpoints in cancer immunotherapy. Nature Reviews Cancer. 2012;12:252.

29. Dong H, Strome SE, Salomao DR, et al. Tumor-associated B7-H1 promotes T-cell apoptosis: A potential mechanism of immune evasion. Nature Medicine. 2002;8:793.

30. Iwai Y, Ishida M, Tanaka Y, Okazaki T, Honjo T, Minato N. Involvement of PD-L1 on tumor cells in the escape from host immune system and tumor immunotherapy by PD-L1 blockade. Proceedings of the National Academy of Sciences. 2002;99(19):12293-12297.

31. Zou W, Chen L. Inhibitory B7-family molecules in the tumour microenvironment. Nature Reviews Immunology. 2008;8:467.

32. Hino R, Kabashima K, Kato Y, et al. Tumor cell expression of programmed cell death-1 ligand 1 is a prognostic factor for malignant melanoma. Cancer. 2010;116(7):1757-1766.

33. Brahmer JR, Tykodi SS, Chow LQM, et al. Safety and Activity of Anti-PD-L1 Antibody in Patients with Advanced Cancer. New England Journal of Medicine. 2012;366(26):2455-2465.

34. Rittmeyer A, Barlesi F, Waterkamp D, et al. Atezolizumab versus docetaxel in patients with previously treated non-small-cell lung cancer (OAK): a phase 3, open-label, multicentre randomised controlled trial. The Lancet. 2017;389(10066):255-265.

35. Plimack ER, Bellmunt J, Gupta S, et al. Safety and activity of pembrolizumab in patients with locally advanced or metastatic urothelial cancer (KEYNOTE-012): a non-randomised, open-label, phase 1b study. Lancet Oncol. 2017;18(2):212-220.

36. Tumeh PC, Hellmann MD, Hamid O, et al. Liver Metastasis and Treatment Outcome with Anti-PD-1 Monoclonal Antibody in Patients with Melanoma and NSCLC. Cancer Immunol Res. 2017;5(5):417-424.

37. Ott PA, Bang YJ, Berton-Rigaud D, et al. Safety and Antitumor Activity of Pembrolizumab in Advanced Programmed Death Ligand 1-Positive Endometrial Cancer: Results From the KEYNOTE-028 Study. J Clin Oncol. 2017;10(10):JCO2017725952.

38. Herbst RS, Baas P, Kim D-W, et al. Pembrolizumab versus docetaxel for previously treated, PD-L1positive, advanced non-small-cell lung cancer (KEYNOTE-010): a randomised controlled trial. The Lancet.387(10027):1540-1550.

39. Reck M, Rodriguez-Abreu D, Robinson AG, et al. Pembrolizumab versus Chemotherapy for PD-L1Positive Non-Small-Cell Lung Cancer. N Engl J Med. 2016;375(19):1823-1833. 
40. Ratcliffe MJ, Sharpe A, Midha A, et al. Agreement between Programmed Cell Death Ligand-1 Diagnostic Assays across Multiple Protein Expression Cutoffs in Non-Small Cell Lung Cancer. Clinical Cancer Research. 2017;23(14):3585-3591.

41. Garon EB, Rizvi NA, Hui R, et al. Pembrolizumab for the Treatment of Non-Small-Cell Lung Cancer. New England Journal of Medicine. 2015;372(21):2018-2028.

42. Skov BG, Høgdall E, Clementsen P, et al. The prevalence of EGFR mutations in non-small cell lung cancer in an unselected Caucasian population. APMIS. 2015;123(2):108-115.

43. McLaughlin J, Han G, Schalper KA, et al. Quantitative assessment of the heterogeneity of pd-11 expression in non-small-cell lung cancer. JAMA Oncology. 2016;2(1):46-54.

44. Thunnissen E, Allen TC, Adam J, et al. Immunohistochemistry of Pulmonary Biomarkers: A Perspective From Members of the Pulmonary Pathology Society. Archives of Pathology \& Laboratory Medicine. 2017.

45. Gaule P, Smithy JW, Toki M, et al. A quantitative comparison of antibodies to programmed cell death 1 ligand 1. JAMA Oncology. 2017;3(2):256-259.

46. Tseng Y-H, Ho H-L, Lai C-R, et al. Brief Report: PD-L1 expression of tumor cells, macrophages, and immune cells in non-small cell lung cancer patients with malignant pleural effusion. Journal of Thoracic Oncology. 2017.

47. Heymann JJ, Bulman WA, Swinarski D, et al. PD-L1 expression in non-small cell lung carcinoma: Comparison among cytology, small biopsy, and surgical resection specimens. Cancer Cytopathology. 2017;125(12):896-907.

48. Skov BG, Skov T. Paired Comparison of PD-L1 Expression on Cytologic and Histologic Specimens From Malignancies in the Lung Assessed With PD-L1 IHC 28-8pharmDx and PD-L1 IHC 22C3pharmDx. Applied Immunohistochemistry \& Molecular Morphology. 2017;25(7):453-459.

49. Ilie M, Juco J, Huang L, Hofman V, Khambata-Ford S, Hofman P. Use of the 22C3 anti-programmed death ligand 1 antibody to determine programmed death ligand 1 expression in cytology samples obtained from non-small cell lung cancer patients. Cancer Cytopathology. 2018.

50. Cedrés S, Ponce-Aix S, Pardo-Aranda N, et al. Analysis of expression of PTEN/PI3K pathway and programmed cell death ligand 1 (PD-L1) in malignant pleural mesothelioma (MPM). Lung Cancer. 2016;96:1-6.

51. Khanna S, Thomas A, Abate-Daga D, et al. Malignant Mesothelioma Effusions Are Infiltrated by CD3+ T Cells Highly Expressing PD-L1 and the PD-L1+ Tumor Cells within These Effusions Are Susceptible to ADCC by the Anti-PD-L1 Antibody Avelumab. Journal of Thoracic Oncology. 2016;11(11):1993-2005.

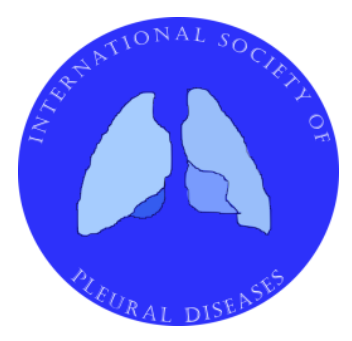

